\title{
The syntax and semantics of negative questions and answers in Korean and English
}

\author{
Keunhyung Park \& Stanley Dubinsky*
}

\begin{abstract}
Differences in Korean and English negative polarity questions (NPQs) are revealed by the interpretation of simple yes-no answers to them. Yes-no answers to NPQs have seemingly unpredictable interpretations (Claus et al. 2017, Holmberg 2013, Kim 2017, Krifka 2017, Kramer \& Rawlins 2009, Ladd 1981, Sudo 2013). However, one clearly observable fact is that yes-no answers to English and Korean NPQs can have opposite interpretations. This study: (i) compares the interpretation of positive and negative polarity questions (PPQs and NPQs) in English and Korean; (ii) examines the structure of negation in each language and its interaction with NPQs, and (iii) reports on an online experiment which gathered native speaker interpretations of NPQs in each language under context-free conditions.
\end{abstract}

Keywords. Semantics; Korean; English; negative polarity questions; proposition external negation (PEN); proposition internal negation (PIN).

1. Polarity questions and answers. English examples (1) and (2) and Korean examples (3) and (4) of polarity questions show that NPQs in these languages, examples (2) and (4), can be interpreted quite differently. We can see this through the ways in which a simple answer of yes or $n o$ is understood.

ENGLISH PPQS AND NPQS

Positive polarity question and answers

(1) Q: Did he eat lunch?

A: Yes, (he did $)=$ 'he ate lunch.' / No, (he didn't) = 'he did not eat lunch.'

Negative polarity question and answers

(2) Q: Didn't he eat lunch?

A: Yes, (he did) = 'he ate lunch.' / No, (he didn't) = 'he did not eat lunch.'

KOREAN PPQS AND NPQS

Positive polarity question and answers

$$
\begin{aligned}
& \text { Q: ku-ka cemsim-ul mekess-ni? } \\
& \text { he-NOM lunch-ACC ate-Q } \\
& \text { 'Did he eat lunch?' } \\
& \text { A: ung, (mekess-e) / ani, (an-mekess-e) } \\
& \text { yes, (ate-DECL) / no, (not-ate-DECL) } \\
& \text { 'Yes, (he ate)' / 'No, (he didn't eat)' }
\end{aligned}
$$

Negative polarity question and answers

$$
\begin{array}{ll}
\mathrm{Q}: & \text { ku-ka cemsim-ul an-mekess-ni? } \\
\text { he-NOM lunch-ACC not-ate-Q } \\
\text { 'Didn't he eat lunch?' }
\end{array}
$$

\footnotetext{
* Authors: Keunhyung Park, University of South Carolina (kp4@email.sc.edu) \& Stanley Dubinsky, University of South Carolina (dubinsky@sc.edu) 


$$
\begin{array}{ccc}
\text { A: ung, (an-mekess-e) } & / & \text { ani, (mekess-e) } \\
\text { yes, (not-ate-DECL) } & / & \text { no, (ate-DECL) } \\
\text { 'Yes, (he didn't eat)' } & / & \text { 'No, (he ate)' }
\end{array}
$$

In both languages, the meaning of a PPQ is straightforward. A yes answer in either English (1) or Korean (3) affirms the assertion that 'he ate lunch', while a no answer denies the assertion. In the case of NPQs, the results are quite different. In the English NPQ (2), a yes response is interpreted in the same manner as a yes reply to the PPQ (1), as affirming that 'he ate lunch'. In Korean, though, yes and no replies to the NPQ (4) are understood in the opposite way, such that a yes reply denies the assertion that 'he ate lunch', while a no response affirms it. How is it, then, that answers to a simple NPQ in English and Korean wind up having opposite interpretations (interpretations that are clearly not obvious from a straightforward literal translation of these sentences)? Do negation in Korean and English and the structure of their NPQs have distinct properties that affect their interpretation?

\section{Proposition external negation (PEN) vs. Proposition internal negation (PIN). Before} moving to syntactic and semantic analysis of NPQs, this section presents descriptive terminology designed to distinguish between uses of negation which are more truth-conditionally relevant and uses of negation that affect the context-dependent pragmatics of a clause. In prior literature on polarity questions, negation in NPQs can be distinguished according to its position in the syntactic structure, and accorded the distinctions of being HIGH, MIDDLE, or LOW (Holmberg 2013). It can also be distinguished, depending on questioner bias, as INNER or OUTER negation (Ladd 1981). Rather than attempt to, a priori, determine the status of negation according to (as yet) uncertain syntactic properties or according to (somewhat subjective) considerations of "questioner bias", we have elected to categorize negatives on the basis of whether they are interpreted as a direct function on the truth of the embedded proposition, or not.

In English examples (1) and (2), we see that yes-no answers to the PPQ/NPQ pair are interpreted identically. The fact that both positive and negative questions have the same interpretation indicates the negation in the NPQ has no effect on the propositional meaning of question. Accordingly, we refer to this category of negation as proposition-external negation (PEN). In contrast, Korean examples (3) and (4) show that the propositional meaning of the NQP is indeed affected by negation, since negation in (4) reverses the truth conditions of (3). We thus refer to this as proposition-internal negation (PIN). While these limited data might seem to indicate that English NPQs have PEN and Korean NPQs have PIN, additional data presented here will reveal that both languages can either PEN and PIN in NPQs, depending in part on the position and type of negation utilized. The next two sections will examine how the positions of negation can affect PEN and PIN interpretations of English and Korean NPQs.

3. The place of negation in English NPQs. A closer look at English, examples (5)-(7), shows that English NPQs can have either PEN and PIN. In (5), negation is outside of VP and undergoes subject-aux inversion (SAI), and the interpretation is clearly PEN. In (6), negation follows a VP adverb and is VP-internal, and the interpretation is clearly PIN. We also find ambiguous cases, such as (7), where negation could be either VP-internal or VP-external, and the interpretation is ambiguously PEN or PIN.

$$
\text { Didn't you eat lunch? }
$$

Yes, I did.

/ No, I didn't.

$(\mathrm{PEN})$

Did you really not eat lunch?

Yes, I didn't.

/ No, I did.

(PIN) 
We can confirm that the negation in (5) is PEN by comparing it to the interpretation options available for a PPQ, here in (8).

Did you eat lunch?

Yes, I did.

/ No, I didn’t.

Seeing that yes and no answers to (5) and (8) are interpreted identically, we can confirm that the negation in (5) does not interact truth-conditionally with the core proposition denoted in the question. Example (5) therefore involves PEN. Conversely, since yes and no answers to (6) are interpreted conversely to those in (8), we can be sure that negation in (6) is indeed interpreted as truth-conditional, and we therefore claim that (6) involves PIN. Example (7) is quite interesting, in comparison with these last two, since a simple yes or no answer to (7) is barely understandable and quite anomalous. In this example, yes and no responses must be augmented with either I did or I didn't, in order for the questioner to make any sense of them. For this reason, we claim that the negation in (7) is ambiguous between PEN and PIN.

4. The place of negation in Korean NPQs. This section examines Korean NPQs, which can variously be interpreted as PEN or PIN depending on the particular negation structure used. Korean clausal negation is formed in two distinct ways. Long-form negation (LFN), in (9), involves nominalization of the verb stem with a $c i$ suffix (e.g. mek-ci 'eat- NMZ) and has the negative prefix directly before a 'do'-support verb $h a$ (e.g. an- $h a$ - 'not-do'). Short-form negation (SFN), seen in (4) and repeated below, involves a negative prefix an attached directly before the verb stem (e.g. an-mek- 'not-eat'). Just as the position of English negation can affect its interpretation (i.e. as PEN or PIN), so too does the choice of negation morphology in Korean correlate differentially with PEN/PIN interpretations. In the LFN construction, where the VP is nominalized by the affix $c i$ and $a$ prefixed to the auxiliary verb $h a$, the negative morpheme occurs outside of the nominalized VP and is interpreted most frequently as PEN (but can be ambiguous between PEN and PIN, with distinctive and contrastive intonation). In the SFN construction, where an 'not' is attached directly before the verb, negation is arguably VP-internal and the interpretation of $a n$ is most consistently (and unsurprisingly) PIN.

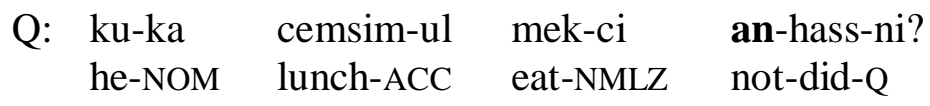

$$
\begin{aligned}
& \text { 'Didn't he eat lunch?' } \\
& \text { A: ung, (mekess-e) / ani, (an-mekess-e) } \\
& \text { yes, (ate-DECL) / no, (not-ate-DECL) } \\
& \text { 'Yes, (he ate)' / 'No, (he didn't eat)' } \\
& \text { A: ung, (an-mekess-e) / ani, (mekess-e) } \\
& \text { yes, (not-ate-DECL) / no, (ate-DECL) } \\
& \text { 'Yes, (he didn't eat)' / 'No, (he ate)' } \\
& \text { (4) Q: ku-ka cemsim-ul an-mekess-ni? } \\
& \text { he-NOM lunch-ACC not-ate-Q } \\
& \text { 'Didn't he eat lunch?' }
\end{aligned}
$$

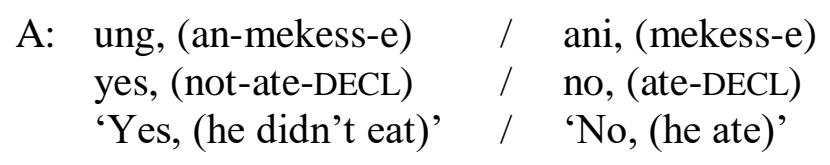


As we have noted, an NPQ with SFN, as in (4), tends to be interpreted as PIN, similar to the English NPQ in (6). We can confirm this by comparing the interpretation of yes-no answers to (4) with yes-no responses to the PPQ in (10). Since ung 'yes' and ani 'no' answers to (4) are interpreted conversely to the ung 'yes' and ani 'no' answers to (10), we can be sure that negation in (4) interacts truth-conditionally with the core proposition and that (4) involves PIN.

$$
\begin{array}{lll}
\text { Q: } & \begin{array}{l}
\text { ku-ka cemsim-ul } \\
\text { he-NOM lunch-ACC } \\
\text { 'Did he eat lunch?' }
\end{array} & \begin{array}{l}
\text { mekess-ni? } \\
\text { ate-Q }
\end{array} \\
\text { A: } \begin{array}{l}
\text { ung, (mekess-e) / } \\
\text { yes, (ate-DECL) / }
\end{array} & \begin{array}{l}
\text { ani, (an-mekess-e) } \\
\text { no, (not-ate-DECL) }
\end{array} \\
\text { 'Yes, (he ate)' } & \text { 'No, (he didn't eat)' }
\end{array}
$$

In contrast, many Korean native speakers interpret an NPQ with LFN as potentially ambiguous between PEN and PIN readings. Syntactically, this may be because the negation in LFN is sometimes high enough to elicit a PEN reading and sometimes low enough for a PIN interpretation. When an NPQ with LFN has a PEN reading, the interpretation of yes-no answers to it are identical to those in PPQ (10) - compare the first set of answers in (9) to those in (10).

5. The semantics of NPQs. In traditional accounts on the grammar of questions (Büring \& Gunlogson 2000, Groenendijk \& Stokhof 1984, Hamblin 1976, Kartunnen 1977, Montague 1973), a PPQ such as (8), repeated here, denotes the set of propositions (i.e. $p, \neg p$ ) that are

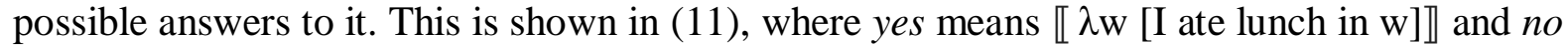
means $\llbracket \lambda w[I$ did not eat lunch in $w] \rrbracket$.

【 Did you eat lunch?

(11) $\{\lambda \mathrm{w}[\mathrm{I}$ ate lunch in $\mathrm{w}], \lambda \mathrm{w}[\mathrm{I}$ did not eat lunch in $\mathrm{w}]\}$

An NPQ having PIN, such as (6), denotes a set in which each proposition is negated $\{\neg p, \neg \neg p\}$, with a yes response meaning $\neg p$ and no meaning $\neg \neg p$ (i.e. $p$ ). In (12), the set of answers to (6) is illustrated, with yes meaning $\llbracket \lambda \mathrm{w}$ [I really did not eat lunch in $\mathrm{w}] \rrbracket$ and no meaning $\llbracket \lambda \mathrm{w}$ [I did not really not eat lunch in w]】. In this view, a yes response to PIN-NPQ has the opposite polarity of a yes answer to a PPQ.

【Did you really not eat lunch? 》

(12) $\{\lambda w$ [I really did not eat lunch in $w], \lambda w$ [I did not really not eat lunch in $w]\}$

When we examine PEN-NPQs, we find that a PEN-NPQ such as (5) denotes the same set of propositions (i.e. $p, \neg p$ ) that are possible answers to a PPQ such as (8). This is shown in (13), where yes means $\llbracket \lambda \mathrm{w}[\mathrm{I}$ ate lunch in $\mathrm{w}] \rrbracket$ and no means $\llbracket \lambda \mathrm{w}[\mathrm{I}$ did not eat lunch in $\mathrm{w}] \rrbracket$.

【Didn't you eat lunch? 》

Thus, because negation is outside the propositional frame, the meaning of PPQs and PEN-NPQs are logically identical, and a "Yes, I did" answer to "Didn't you eat?" and to "Did you eat?" can often both mean the same thing. The difference between (5) and (8), then, results from the PEN negation being pragmatically connected to speaker expectations (Ladd 1981). One plausible distinction, suggested in Romeo \& Han 2003, is that the preposed negation in (5) carries an 
implicature that the questioner believes a priori that 'you did eat lunch', one which is not available in the PPQ (8), nor in the PIN-NPQ (6).

6. Context-free interpretation of NPQs: Experimental design. This section describes a series of online experiments designed to test native Korean and English speakers' processing of yes-no questions. These experiments were explicitly constructed to find out, as accurately as possible, how different types of NPQs are interpreted in the absence of context. The questions that our experiments were designed to address are:

1. How are differently constructed NPQs interpreted without context?

2. Does the interpretation of NPQs correlate predictably with the position of negation relative to the VP and with the PEN/PIN dichotomy?

3. Are there language-particular differences in native-speaker interpretation of NPQs that are independent of structure?

We conducted a series of three on-line experiments for each language. These were conducted using DMDX Display Software ${ }^{i}$ to monitor participants' response times (RT) and error rates (ER).

Each question was composed of two successive slides: 1. a slide displaying a symbol, and 2. a slide displaying a written question with answer choices. The first slide displayed, for 1000ms, one of five different symbols (e.g. triangle, star, square, $X$, and circle) in one of five different colors (e.g. red, blue, black, yellow, and green). The second (Q/A) slide presented a question about the first slide. On this slide, one of the 48 target or 24 filler questions was randomly presented for $5000 \mathrm{~ms}$. While the Q/A slide was shown, participants choose their answer by pressing the right shift-key for yes (or a color/shape term) or the left shift-key for no (or a color/shape term). Following the Q/A slide, a new symbol and Q/A slide pair were presented, repeated this process until the experiment ended.

The English and Korean target questions consisted of PPQs, PEN-NPQs, and PIN-NPQs, as illustrated here in (14) and (15).
a. Did you see a red circle?
b. Didn't you see a red circle?
(PPQ)
c. Did you really not see a red circle?
(PEN-NPQ)
(PIN-NPQ)
a. ppalkan wen-ul po-ass-na-yo?
red circle-ACC see-PST-Q-HON
(PPQ)
'Did you see a red circle?'
b. ppalkan wen-ul po-ci an-hass-na-yo?
red circle-ACC see-NMLZ not-did-Q-HON
'Didn't you see a red circle?'
c. ppalkan wen-ul an-po-ass-na-yo?
red circle-ACC not-see-PST-Q-HON
(PEN-NPQ)
'Didn't you see a red circle?'
(PIN-NPQ)

In each experiment, the 48 target questions exemplified in (14) and (15) were mixed with 24 wh and tag filler questions, exemplified in (16). 

a. What color was the symbol you saw?
b. What shape was the symbol you saw?
c. You saw a red circle, didn't you?

Since the experiments were intended to explore subjects' context-free interpretations of NPQs, several measures were taken to insure that subjects were not exposed to pragmatic cues. First, the visual prompts (the randomly shaped and differently colored symbols) were displayed without any context or background - just the symbol on an otherwise blank screen. Target and filler questions were also mixed randomly, as was the likelihood of the correct answer to a target or tag question being yes or no. Finally, questions were presented in written form, to insure that subjects would not be exposed to meta-linguistic pragmatic cues, such as stress or intonation.

The experimental series for each language was divided into three individual experiments, where each experiment manipulated one of the following contrasts:

- Experiment 1 tested PPQs vs. PEN-NPQs

- Experiment 2 tested PPQs vs. PIN-NPQs

- Experiment 3 tested PPQs vs. PEN-NPQs vs. PIN-NPQs.

Subjects were tested on data from their own native language only, and no subject took part in more than one of the three experiments. Experiments 1 and 2 presented each subject with 24 PPQs and 24 NPQs, and Experiment 3 presented subjects with 24 PPQs, 24 PEN-NPQs, and 24 PIN-NPQs. Each set of 24 target questions was divided into 12 designed to induce yes-answers and 12 designed to induce no-answers. These target stimuli were randomly mixed with 24 filler questions (e.g. 12 wh-questions and 12 tag questions). Korean Experiment 1 had 7 participants, Korean Experiment 2 had 6 participants, and Korean Experiment 3 had 6 participants. English Experiment 1 had 4 participants, English Experiment 2 had 5 participants, and English Experiment 3 had 4 participants.

7. Experimental results. To ascertain subjects' interpretations of NPQs, we measured subjects' response times (RT) and error rates (ER) when tested on the target questions. Overall results showed there to be significant differences between PPQs and NPQs in all three experiments. Moreover, significant RT and ER differences in responses to PEN-NPQs and PIN-NPQs were also recorded. Differential responses by these categories were parallel for Korean and English.

7. 1. RESPONSE TIME (RT). English and Korean participants had longer RTs for NPQs than for PPQs in Exp 1 and 2. Also, in Exp 3, which presented both PEN-NPQs and PIN-NPQs along with PPQs, NPQs as a class had longer RTs than PPQs. Also, in Exp 3, RTs for PIN-NPQs were longer than for PEN-NPQs (significantly so in Exp 3-E). The results are summarized below:

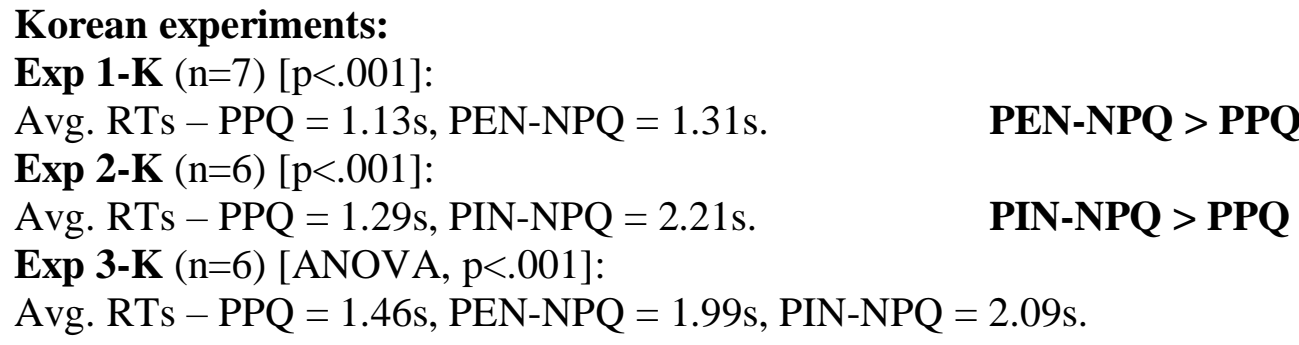




\section{English experiments:}

$\operatorname{Exp} 1-\mathbf{E}(\mathrm{n}=4)[\mathrm{p}<.01]:$

Avg. RTs $-\mathrm{PPQ}=1.12 \mathrm{~s}, \mathrm{PEN}-\mathrm{NPQ}=1.36 \mathrm{~s}$.

$\operatorname{Exp} 2-\mathbf{E}(n=5)[p<.001]$ :

Avg. RTs $-\mathrm{PPQ}=1.21 \mathrm{~s}, \mathrm{PIN}-\mathrm{NPQ}=2.37 \mathrm{~s}$.

PEN-NPQ > PPQ

Exp 3-E ( $n=4)$ [ANOVA, p<.001]:

PIN-NPQ > PPQ

Avg. RTs $-\mathrm{PPQ}=1.29 \mathrm{~s}, \mathrm{PEN}-\mathrm{NPQ}=1.57 \mathrm{~s}, \mathrm{PIN}-\mathrm{NPQ}=2.30 \mathrm{~s}$.

PIN-NPQ > PEN-NPQ > PPQ

7. 2. ERROR RATE (ER). The ERs for all target questions were collected in tandem with RTs. Before presenting the ER results, we must clarify what we mean by errors. ERs for PPQs are quite straightforward, since the answer to Did you see a red circle? following the presentation of a red circle is correct for yes and incorrect for no. Contrariwise, following the presentation of a green triangle, yes is incorrect and no is correct. With NPQs, though, which are normally subject to a high degree of context-driven ambiguity (based on speaker and hearer expectations), the calculation of ERs is somewhat more nuanced. PEN-NPQs do not typically alter truthconditional meaning, and so we considered correct answers to these (in the absence of any overt context) to be identical to those for PPQs. Since PIN-NPQs do indeed reverse the truthconditional meaning of their corresponding PPQ, we considered correct answers to these (in the absence of context) to be the reverse of their corresponding PPQ. Table 1 below shows the percentage and number of errors by question type in three experiments.

\begin{tabular}{llccc} 
& & Exp 1 \& & Exp 3 & All Experiments \\
\hline \multirow{3}{*}{ Korean } & PPQ & $2.9 \%(9 / 312)$ & $3.5 \%(5 / 144)$ & $3.1 \%(14 / 456)$ \\
& PEN-NPQ & $1.2 \%(2 / 168)$ & $20.1 \%(29 / 144)$ & $9.9 \%(31 / 312)$ \\
& PIN-NPQ & $16.7 \%(24 / 144)$ & $36.1 \%(52 / 144)$ & $26.4 \%(76 / 288)$ \\
\hline \multirow{3}{*}{ English } & PPQ & $3.7 \%(8 / 216)$ & $4.2 \%(4 / 96)$ & $3.9 \%(12 / 312)$ \\
& PEN-NPQ & $6.3 \%(6 / 96)$ & $10.4 \%(10 / 96)$ & $8.3 \%(16 / 192)$ \\
& PIN-NPQ & $34.2 \%(41 / 120)$ & $27.1 \%(26 / 96)$ & $31.0 \%(67 / 216)$ \\
\hline
\end{tabular}

Table 1. The percentage and number of errors for the three experiments

In Exp 1 and 2, ERs for Korean and English PPQs were about 3\% and 4\%, respectively, and ERs for Korean and English PEN-NPQs were about $1 \%$ and $6 \%$. These results, combined with shorter RTs for PEN-NPQs, suggests that participants did not consider negation in calculating the truthvalue of their answers. In contrast, ERs for Korean and English PIN-NPQs were about 17\% and $34 \%$, respectively. This, together with the longer RTs for PIN-NPQs, suggests that speakers did attempt to include negation in calculating the truth-value of their answers, and that this was perhaps confusing in a context-free environment. In Exp 3, the number of errors is somewhat different from the errors in Exp 1 and 2. Nevertheless, in both English and Korean, participants commonly committed more errors in PIN-NPQs than in PPQs or PEN-NPQs. This tendency was also maintained when errors in Exp 1, 2, and 3 were summed together.

8. Discussion and conclusions. RTs and ERs from Exp 1 (PPQ vs. PEN-NPQ), Exp 2 (PPQ vs. PIN-NPQ), and Exp 3 (PPQ vs. PEN-NPQ vs. PIN-NPQ) reveal that participants responded differently, and significantly so, to each question type. As previously suggested, answering a PPQ is very straightforward and does not invoke any special processing cost in choosing between yes and no answers. However, when negation is involved (in the two types of NPQs), it induces semantic and pragmatic processing costs (along with some degree of uncertainty) which 
correlate to some extent with the position of negation in the NPQ. The PEN-PIN dichotomy clearly led to significant differences in RTs and ERs in the current experiments. With these results in hand, we return to the questions posed earlier.

How are differently constructed NPQs interpreted without context? Ladd 1981 first noted that NPQs with same structure can have distinct interpretations depending on the conversational context of their use and speaker expectations. Assuming context to be critical to the pragmatic interpretation of NPQs and determined to explore their semantic meaning, we tested subjects' interpretation of these in a situation that offered no contextual or pragmatic cues. This was done by presenting and asking about differently colored and shaped symbols and by presenting target questions in written form. The results demonstrate that NPQs without any context are still inherently more difficult to interpret than PPQs, as confirmed by longer RTs and higher ERs.

Does the interpretation of NPQs correlate predictably with the position of negation relative to the VP and with the PEN/PIN dichotomy? Both English and Korean allow negation to appear in different positions (high vs. low), and these different positions appear to correlate with their being interpreted either outside or inside the VP. In English, negation in an NPQ can undergo SAI and appear in the left periphery. Alternatively, NPQ negation can appear adjacent to the verb and inside the scope of proposition-oriented or agent-oriented adverbs. In the first case, negation is not calculated into the truth-functional interpretation of the proposition (PEN), and in the second case it is (PIN). Korean, likewise, can have NPQ negation attached high in the clause and outside the scope of a nominalized VP, or directly affixed to the verb inside the VP. Similar to English, the structure having high negation (LFN) tends not to have that negation calculated into the interpretation of the core proposition (PEN), and the structure with low negation (SFN) does affect the propositional meaning directly (PIN).

RTs and ERs were higher for PEN-NPQs than for PPQs. Since the PEN-NPQ and PPQ have essentially the same truth-conditions, the difficulty in processing the former might have to do with (i) the fact that PEN-NPQs have more complex structure (i.e., they include negation), and (ii) the expectation that PEN-NPQ negation is used to transmit pragmatic, conversational cues and such cues were entirely absent from the experiment. Accordingly, subjects had to (i) process the negation in the structure (absent in PPQs), and (ii) determine that it should be disregarded (there being no contextual basis for calculating conversational implicatures from its use). Thus, subjects took longer to process these target questions, and made more errors.

RTs and ERs were even higher for PIN-NPQs than for both PEN-NPQs and PPQs. In these cases, subjects still had to process the more complex structure (one involving negation). However, rather than simply determining that negation was not relevant to interpretation, they had to actually calculate the semantic consequences of negating the core proposition. Hence, they took even longer to process and interpret these target questions, and made even more errors.

Lastly, are there language-particular differences in native-speaker interpretation of NPQs that are independent of structure? We noted at the outset that English and Korean NPQs can have opposite interpretations, based on the meaning of yes and no responses to them (cf. examples (2) and (4)). However, upon further examination of their structures and in consideration of our experimental results, we find their patterns of interpretation to be quite parallel. In both languages, high negation in NPQs does not enter into truth-conditional interpretation, and low negation in NPQs does do so.

The most salient difference between English and Korean appears to be that English negation can appear in a wider variety of positions (each of which having a somewhat different scope from the others), while Korean negation is restricted to exactly two different structures. It may be 
the case that the more varied negation options in English lend themselves to a different conversational calculus than those of Korean. However, an exploration of these factors would necessarily need experiments that reintroduce them into conversational contexts and explore the world of conversationally induced implicatures and speaker expectations. Such research is clearly needed, and would be usefully informed by the decontextualized baseline experiments that we report here.

\section{References}

Büring, Daniel \& Christine Gunslogson. 2000. Aren't positive and negative polar questions the same? Manuscript. University of California, Santa Cruz.

Claus, Berry, A. Meijer Marlijn, Sophie Repp \& Manfred Krifka. 2017. Puzzling response particles: An experimental study on the German answering system. Semantics and Pragmatics 10(19). 1-51. https://doi.org/10.3765/sp.10.19.

Groenendijk, Jeroen. \& Martin Stokhof. 1984. Studies on the semantics of questions and the pragmatics of answers. University of Amsterdam dissertation.

Hamblin, Charles L. 1973. Questions in Montague English. Foundations of Language 10. 41-53.

Holmberg, Anders. 2013. The syntax of answers to polar questions in English and Swedish. Lingua 128: 31-50. https://doi.org/10.1016/j.lingua.2012.10.018.

Karttunen, Lauri. 1977. Syntax and semantics of questions. Linguistics and Philosophy 1(1). 344. https://doi.org/10.1007/BF00351935.

Kim, Jong-Bok. 2017. On the anaphoric nature of particle responses to the polar questions in English and Korean. Korean Journal of Linguistics 42(2). 153-177.

Kramer, Ruth, and Kyle Rawlins. 2011. Polarity particles: An ellipsis account. Proceedings of the $39^{\text {th }}$ Meeting of the Northeast Linguistics Society (NELS) 39. 479-492.

Krifka, Manfred. 2017. Negated polarity questions as denegations of assertions. In Chungmin Lee, Ferenc Kiefer, \& Manfred Krifka (eds.), Contrastiveness in information structure, alternatives, and scalar implicatures. 359-398. Dordrecht: Springer.

Ladd, D. Robert. 1981. A first look at the semantics and pragmatics of negative questions and tag questions. Papers from the Seventeenth Regional Meeting of the Chicago Linguistic Society (CLS) 17. 164-171.

Montague, Richard. 1973. The proper treatment of quantification in ordinary English. In Patrick Suppes, Julius Moravcsik \& Jaakko Hintikka (eds.), Approaches to natural language:

Proceedings of the 1970 Stanford Workshop on Grammar and Semantics. 221-242. Dordrecht: Springer.

Romero, Maribel \& Chung-Hye Han. 2004. On negative yes/no questions. Linguistics and Philosophy, 27(5). 609-658.

Sudo, Yasutada. 2013. Biased polar questions in English and Japanese. In Daniel Gutzmann \& Hans-Martin Gaertner (eds.). Beyond expressives: Explorations in use-conditional meaning . 275-295. Leiden: Brill.

\footnotetext{
${ }^{1}$ DMDX is a Win 32-based display system used in psychological laboratories round the world to measure reaction times to visual and auditory stimuli. It was programmed by Jonathan Forster at the University of Arizona

[http://www.u.arizona.edu/ kforster/dmdx/dmdx.htm].
} 